\title{
The utility of positron emission tomography in staging of potentially operable carcinoma of the thoracic esophagus: Results of the American College of Surgeons Oncology Group Z0060 trial
}

Bryan F. Meyers, MD, MPH, ${ }^{a}$ Robert J. Downey, MD, ${ }^{c}$ Paul A. Decker, MS, ${ }^{d}$ Robert J. Keenan, MD, ${ }^{\text {}}$ Barry A. Siegel, MD, ${ }^{b}$ Robert J. Cerfolio, MD, ${ }^{f}$ Rodney J. Landreneau, MD, ${ }^{\mathrm{g}}$ Carolyn E. Reed, MD, ${ }^{\mathrm{h}}$ Dennis M. Balfe, MD, Farrokh Dehdashti, MD, ${ }^{\mathrm{b}}$ Karla V. Ballman, PhD, ${ }^{d}$ Valerie W. Rusch, MD, ${ }^{\mathrm{c}}$ and Joe B. Putnam, Jr, MD ${ }^{\mathrm{i}}$

Earn CME credits at http:// cme.ctsnetjournals.org

๑ Supplemental material is available online.

From the Departments of Surgery and Radiology, ${ }^{\text {b }}$ Washington University School of Medicine, St. Louis, Mo; Department of Surgery, ${ }^{\mathrm{c}}$ Memorial Sloan-Kettering Cancer Center, New York, NY; Department of Health Sciences Research, ${ }^{\mathrm{d}}$ Mayo Clinic College of Medicine, Rochester, Minn; Department of Surgery, ${ }^{\mathrm{e}}$ Allegheny General Hospital, Pittsburgh, Pa; Department of Cardiothoracic Surgery, ${ }^{\mathrm{f}}$ University of Alabama at Birmingham, Ala; Department of Surgery, ${ }^{\mathrm{g}}$ University of Pittsburgh, Pa; Department of Cardiothoracic Surgery, ${ }^{\mathrm{h}}$ Medical University of South Carolina, Charleston, SC; and Department of Thoracic Surgery, ${ }^{i}$ Vanderbilt University Medical Center, Nashville, Tenn.

Barry Siegel reports consulting or lecture fees from Cardinal Health Pet/Net, DMS Imaging, GE/Phillips, Radiology Corporation of America (RCOA), RCOA/Siemens, and Spectrum Dynamics and equity ownership in RCOA.

Read at the Eighty-sixth Annual Meeting of The American Association for Thoracic Surgery, Philadelphia, Pa, April 29-May 3, 2006.

Received for publication April 28, 2006; revisions received Aug 9, 2006; accepted for publication Sept 26, 2006.

Address for reprints: Bryan F. Meyers, MD, MPH, Division of Cardiothoracic Surgery, Washington University School of Medicine, Suite 3108 Queeny Tower, 1 BarnesJewish Hospital Plaza, St Louis, MO 63110 (E-mail: meyersb@wustl.edu).

J Thorac Cardiovasc Surg 2007;133:738-45 $0022-5223 / \$ 32.00$

Copyright () 2007 by The American Association for Thoracic Surgery

doi:10.1016/j.jtcvs.2006.09.079
Objectives: The American College of Surgeons Oncology Group trial Z0060 is a prospective multi-institutional trial with a primary objective to evaluate whether positron emission tomography (PET) with F-18 fluorodeoxyglucose (FDG) detects evidence of metastastic disease that precludes esophagectomy in patients with esophageal cancer who are surgical candidates after routine staging.

Methods: Patients with resectable, biopsy-proven carcinoma were enrolled after computed tomography of chest and abdomen demonstrated no evidence of metastasis. FDG-PET was performed according to specified standards. FDG-PET findings suggesting metastases required confirmation and patients without metastases on PET were expected to proceed to surgery.

Results: A total of 262 patients were registered. Of these, 199 were deemed eligible and of these, 189 patients were evaluable. Seventy-three patients were ineligible or unevaluable. Reasons for ineligibility included nonresectable disease by routine staging (39), missing or outdated staging procedures (12), PET technical protocol violations (10), no cancer (4), pre-PET induction therapy (3), claustrophobia (1), and other causes (4). There were 145 (78\%) patients who went on to have surgery, $42(22 \%)$ who did not, and 2 patients for whom the surgical status was not determined. The reasons for no resection included the following: M1 disease found by PET and confirmed (9), M1 disease found by PET and not confirmed (2), M1 disease at exploration not found by PET (7), decline or death before surgery (10), patient refusal of surgery (7), unresectable local tumor at exploration (5), and extensive N1 disease precluding operation (2). Eight (4.2\%) patients undergoing resection had a recurrence in the first 6 months.

Conclusions: Although $22 \%$ of eligible patients did not undergo esophagectomy, FDG-PET after standard clinical staging for esophageal carcinoma identified confirmed M1b disease in at least $4.8 \%$ (95\% confidence interval: $2.2 \%-8.9 \%$ ) of patients before resection. Unconfirmed PET evidence of M1 disease and regional adenopathy (N1 disease) led to definitive nonsurgical or induction therapy in additional patients.

A ccurate staging of esophageal cancer is essential to the ability to offer patients treatment and an estimate of prognosis; it also allows adequate interpretation and comparison of research studies. A significant advance in the staging of esophageal cancer has been the use of 18F-fluorodeoxyglucose positron emission tomography (FDG-PET). This imaging modality takes advantage of the fact that cancer cells have an increased cellular uptake of glucose and higher rate of glycolysis than do normal cells. The radiolabeled glucose analog $\left[{ }^{18} \mathrm{~F}\right]$ fluoro- 


\section{Abbreviations and Acronyms \\ ACOSOG $=$ American College of Surgeons Oncology Group \\ $\mathrm{CI}=$ confidence interval \\ CT = computed tomography \\ FDG $=18$ F-fluorodeoxyglucose \\ FDG-PET $=18$ F-fluorodeoxyglucose positron emission tomography \\ MRI = magnetic resonance imaging \\ PET = positron emission tomography}

2-deoxy-D-glucose undergoes the same cellular uptake as glucose, but, after phosphorylation, it is not further metabolized and is thus trapped in cells. PET, therefore, provides information on the functional activity of a malignant lesion rather than the strictly anatomic information that computed tomography $(\mathrm{CT})$ provides.

Preliminary experience with FDG-PET in 36 patients with esophageal cancer was reported in 1997. ${ }^{1}$ Metastatic disease was suspected in 7 (24\%) of 29 patients who underwent esophagectomy with curative intent, and therefore they underwent limited (confirmatory) tissue sampling rather than resection. In 5 of these 7 patients, PET was able to detect the metastatic focus whereas CT failed to reveal it. In a later update, 58 patients with esophageal cancer were evaluated by FDG-PET. In 52 patients who were deemed clinically operable, 17 were found by PET to have distant metastatic disease that precluded resection, whereas CT detected metastases in only 5 of the 17 patients. ${ }^{2}$ A similar study reported on 50 patients with esophageal cancer who were evaluated by FDG-PET. ${ }^{3}$ Thirty-five patients were considered to have resectable disease by clinical evaluation. However, PET revealed increased FDG uptake beyond the primary tumor in 18 patients. Sixteen of these 18 patients were found to have true positive findings, which included both locoregional and distant metastatic disease. In particular, PET identified distant metastatic spread in 9 of the 18 patients, whereas CT failed to detect distant spread in any of these 9 patients. The use of PET for the detection of distant metastases has been insufficiently studied, although reports have documented unexpected metastases in about $10 \%$ of patients who are otherwise deemed candidates for surgery. ${ }^{4}$ One consistent observation with PET staging of esophageal cancer is that PET appears to be more sensitive than CT in identifying distant metastatic spread. In most of the above reports, the majority of positive PET findings were validated by confirmatory tissue biopsies. This is clearly beneficial in that it spares these patients from undergoing an unnecessary and noncurative esophagectomy. Unfortunately, the preliminary data either involve small numbers of patients or else reflect studies that are methodologically sub- optimal. In addition, there are no definitive data concerning false positive PET findings.

To clarify the role of FDG-PET in the staging potentially resectable esophageal cancer, the American College of Surgeons Oncology Group (ACOSOG) undertook the prospective multi-institutional trial Z0060. The primary objective was to ascertain whether FDG-PET could detect metastatic lesions that would preclude esophageal resection in patients believed to be surgical candidates after standard imaging procedures.

\section{Materials and Methods \\ Regulatory Requirements}

Investigators registering patients to Z0060 were required to be members in good standing of ACOSOG, and the clinical site had to be affiliated with an active ACOSOG institution. The Z0060 protocol underwent institutional review board approval at all clinical sites. Before enrolling patients in the study, the nuclear medicine investigator at participating institutions was required to submit the detailed institutional protocol for PET imaging for Z0060 along with images and reports from three consecutive PET studies for evaluation and approval by the ACOSOG PET Quality Assurance Committee. The protocol was initially activated on November 22, 1999. The first patient was enrolled on February 15, 2000, and the last patient was enrolled on July 10, 2004.

\section{Inclusion Criteria}

Patients older than 18 years of age with histologically confirmed adenocarcinoma or squamous cell carcinoma of the esophagus or the gastroesophageal junction were considered eligible. The tumors had to be at least $20 \mathrm{~cm}$ from the incisors and the patients had to be free of metastatic disease after clinical and radiologic screening. Acceptable stages included T1-3 N0-1 M0-1a, and patients had to be medically fit for surgical staging and esophagectomy. Patients with poorly controlled diabetes mellitus were excluded, as were those unable to tolerate PET and those with a previous PET. Patients in whom neoadjuvant chemotherapy or chemoradiotherapy was planned were excluded initially, but concerns about enrollment led to a 2001 protocol amendment that allowed the inclusion of such patients if PET was performed before neoadjuvant therapy. Thirty-seven (19.6\%) patients were enrolled before the amendment and $152(80.4 \%)$ were enrolled after the amendment. Survivors of previous cancers were eligible only if they had undergone curative therapy and had been free of recurrence for at least 5 years. It became apparent after enrollment of many patients that the declaration of "free of metastatic disease" was subjective and many subjects enrolled had findings on CT scan that would be considered evidence of metastases. Noncalcified lung lesions $4 \mathrm{~mm}$ or less were acceptable, whereas larger lesions were considered suggestive of metastases. Liver abnormalities described as cysts or likely cysts were accepted, whereas lesions described as suggestive of metastases were not. Other lesions that were described by the initial radiologist as suggestive of metastasis were similarly excluded for analysis of the primary end point. 


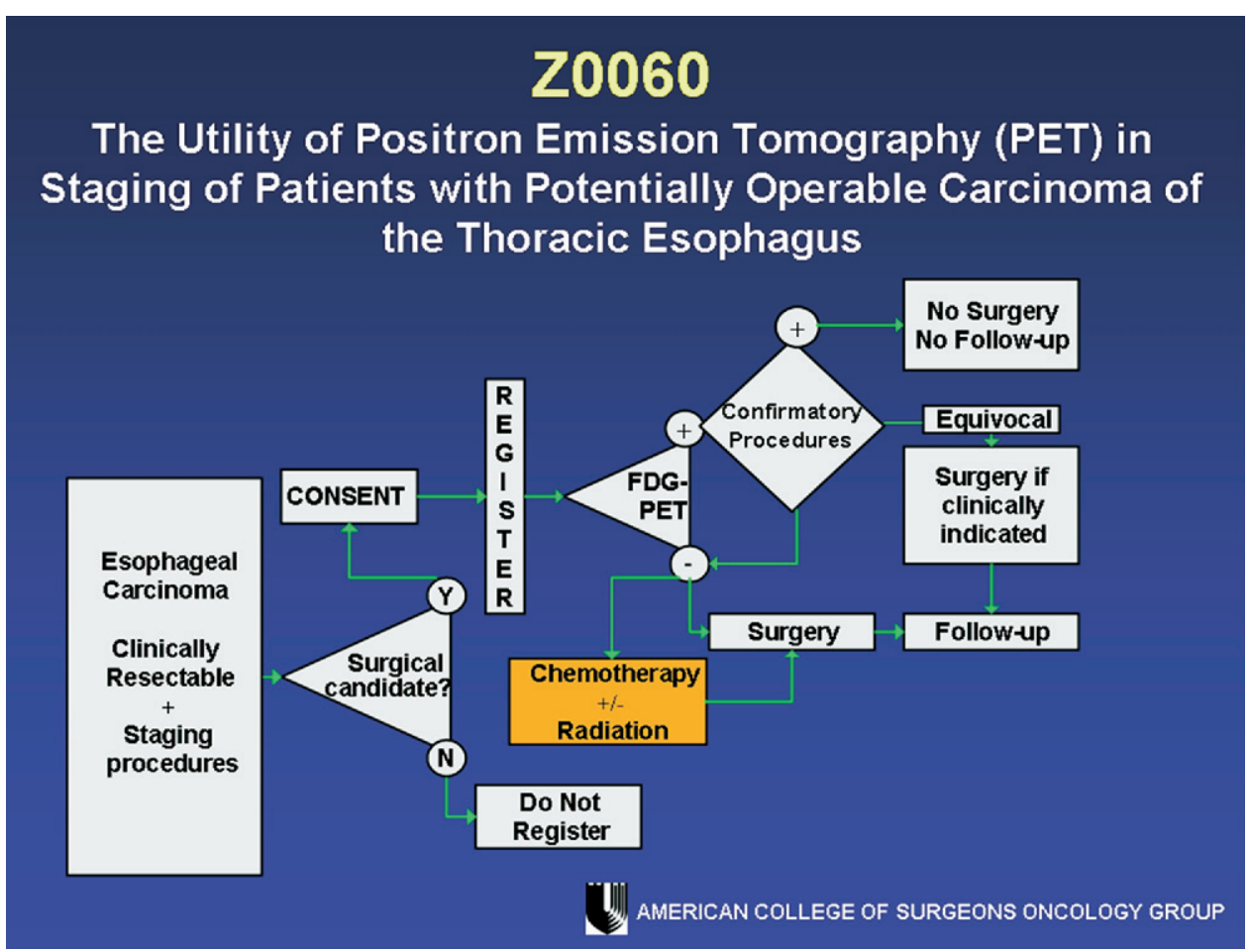

Figure 1. The Z0060 study schema.

\section{Radiologic Assessments}

Mandatory radiologic staging assessments included chest and abdominal CT. Bone scintigraphy and CT or magnetic resonance imaging (MRI) of the brain were required only if dictated by clinical signs, symptoms, or abnormal laboratory results. Radiologic assessments were required to occur within 30 days before study enrollment. CT from the thoracic inlet to the inferior tip of the liver with intravenous contrast administration (unless contraindicated) was performed with fourth-generation scanners capable of high resolution, wide-dynamic range, and rapid screening. Collimation did not exceed $5 \mathrm{~mm}$ and the spacing between slice centers was no greater than $8 \mathrm{~mm}$.

\section{Clinical Assessment}

Clinical assessment was performed 30 days before registration and included an extended history and physical examination.

\section{Study Schema}

Patients whose disease was clinically staged as T1-3 N0-1 M0-1a after standard staging procedures and who were surgical candidates were then registered to Z0060. Figure 1 illustrates the study schema.

\section{FDG-PET}

Full-ring dedicated scanners with bismuth germanate or sodium iodide detectors and with manufacturer-quoted in-plane spatial resolution of less than $6 \mathrm{~mm}$ were used. Imaging with hybrid PET/CT scanners, which became commercially available in 2002, was not allowed in this study. The scanners underwent quality control evaluation on each day imaging was performed. Patients fasted for at least 4 hours before the PET study, but were well hydrated. The blood glucose concentration was determined immediately before FDG administration, and the study was not performed if the blood glucose concentration was greater than 200 $\mathrm{mg} / \mathrm{dL}$. FDG was obtained from a commercial supplier or was prepared and quality controlled by the routine method in use at the performance site in a manner consistent with applicable state and federal regulations. The radiochemical purity of the FDG was required to be greater than $90 \%$. For facilities with a dedicated bismuth germanate PET system, the FDG dose was 0.14 to 0.21 $\mathrm{mCi} / \mathrm{kg}$, with a minimum dose of $10 \mathrm{mCi}$. For facilities with a sodium iodide PET system, a dose of $0.07 \mathrm{mCi} / \mathrm{kg}$ was used.

PET imaging of the body began 45 to 60 minutes after FDG injection. The patient was positioned supine, with the arms comfortably positioned above the head, whenever possible. The region imaged extended from the upper/mid neck to the upper thigh. A series of 5 or 6 overlapping transmission scans were obtained with a rotating ${ }^{68} \mathrm{Ge} /{ }^{68} \mathrm{Ga}$ rod source for each emission scan. A segmentation algorithm was used for all transmission scans, with scan duration of 2 to 3 minutes. With dedicated sodium iodide PET systems, attenuation correction was done with a Cs-137 source in accordance with the manufacturer's recommendations. Then, 5 or 6 corresponding emission images were performed. The PET images were reconstructed both with and without attenuation correction by standard vendor-provided reconstruction algorithms, using either filtered back projection with a Hann filter (frequency cutoff $0.6 \times$ Nyquist $=0.3$ cycles/pixel) or an iterative reconstruction method with an appropriate filter. Segmentation of transmission images was used for attenuation correction. Emission data were corrected for random coincidences and dead-time. The conven- 
tional and PET imaging studies from the first 3 patients at each site and a $20 \%$ random sample of subsequent patients were reviewed by 3 radiology/nuclear medicine investigators to document that these studies were performed per protocol specifications.

The PET images were interpreted by an experienced nuclear physician from each participating site. The transmission data were reviewed with the emission scans to facilitate anatomic localization of abnormal activity. The scans were reviewed in axial, coronal, and sagittal planes and as maximum-pixel-intensity reprojection images. The images were initially interpreted without knowledge of the results of previously obtained CT or other imaging studies or of surgical staging procedures. The images were then reinterpreted in combination with the CT and other available imaging studies. These "unblinded" readings, which most closely reproduce routine clinical practice, were used for the primary end point analysis. Visual analysis involved the identification of abnormal uptake as being greater than normal activity on the attenuation-corrected images. The interpreter's degree of suspicion that tumor was present at the primary tumor site, in locoregional nodes, and at distant metastatic sites was recorded on a standard case report form.

Abnormalities by PET that suggested metastases were required to be confirmed by additional studies or biopsies. A PET-positive hepatic lesion required biopsy or fine-needle aspiration cytology to confirm metastatic disease. Benign cysts or hemangiomas could be confirmed by MRI or ultrasonography. PET-positive adrenal lesions required biopsy. Osseous abnormalities seen on PET had to be evaluated by appropriate imaging studies (radiographs, CT, MRI, or repeat bone scintigraphy), by biopsy, or both. In the case of multiple PET-positive lesions, a single positive biopsy specimen of the most technically accessible site was considered satisfactory confirmation of positivity. In many instances, the PET-positive lesions were best accessed by laparotomy or thoracotomy, and the effort to confirm the truth of a PET-detected metastasis took place at the time of the operation, but before actual resection of the esophagus.

When PET did not identify distant metastasis, or when the confirmatory tests suggested or proved a false positive PET result, the protocol intended that the patient would undergo surgical resection with or without induction chemoradiotherapy. Resections without induction therapy were to take place within 30 days of PET. No specific type of resection was required or suggested, and the details of surgical and perioperative care were left to the treating surgeon. Perioperative events were captured and follow-up at 6 months was required to document whether the patient was alive and whether there was evidence of recurrence.

\section{Statistical Methods}

The study was designed to assess the utility of FDG-PET using data from a clinical construct where the standard staging procedures had to be performed and interpreted before the use of PET. The hypothesis associated with the primary objective was as follows: $H_{O}: P=P_{O}$ against $H_{A}: P \geq P_{A}$, where $\mathrm{H}_{0}$ is the null hypothesis, $\mathrm{H}_{\mathrm{A}}$ is the alternative hypothesis, $\mathrm{P}$ is a measure of the utility of PET (surgery contraindicated by PET after negative findings using standard staging), $\mathrm{P}_{\mathrm{O}}$ is the value of $\mathrm{P}$ regarded as clinically inconsequential, and $\mathrm{P}_{\mathrm{A}}$ is the value of $\mathrm{P}$ regarded as clinically consequential. The values for $\mathrm{P}_{0}$ and $\mathrm{P}_{\mathrm{A}}$ were set to be
0.05 and 0.1 , respectively. A 2-stage accrual design was used to allow for early termination if there was strong early evidence to support the null hypothesis. ${ }^{5}$ If the first stage of accrual consisting of 120 patients did not support early suspension, a second stage of accrual of 115 patients for a total of 235 patients was targeted as the final accrual goal. The decision rule for the first stage was to terminate the study if 5 cases or fewer were observed that contraindicated surgery. If 6 or more were observed, the study was to go on to stage 2 . If there were fewer than 18 cases among the first 235 accrued cases where FDG-PET findings contraindicated surgery, then the study will have provided evidence in favor of the null hypothesis. If 18 or more cases of 235 demonstrated FDG-PET findings contraindicating surgery, then the study will have provided evidence in favor of the alternative hypothesis. Point estimates and $95 \%$ exact confidence intervals (CI) were calculated as appropriate. Resection rates were compared between those patients receiving and not receiving induction therapy using a $\chi^{2}$ test.

\section{Results}

From February 2000 to July 2004, 262 patients were registered to Z0060 from 23 institutions (Table E-1). Of the registered patients, 199 were deemed eligible and, of these, 189 patients were evaluable. Sixty-three patients were declared ineligible and 10 were considered eligible but not evaluable. The reasons for ineligibility included nonresectable disease documented by routine staging (39), missing or outdated staging procedures (12), no cancer diagnosis (4), pre-PET induction therapy (3), claustrophobia (1), and other causes (4). The 10 eligible patients excluded for PET protocol violations included 3 who had induction after PET before it was allowed in protocol revisions, 3 who had PET/CT, 2 who died or withdrew before the PET was done, and 2 with specific PET scanning protocol violations.

The mean age $( \pm \mathrm{SD})$ of the 189 evaluable patients was $62.6 \pm 11.0$ years (range: $36.2-88.5$ years); $160(84.7 \%)$ were men and 29 (15.3\%) were women; 180 (95.2\%) were white. The Eastern Cooperative Oncology Group/Zubrod performance status was as follows: 0, $116(61.4 \%) ; 1,71$ (37.6\%); and 2, $2(1.1 \%)$. Thirty-seven (19.6\%) patients were enrolled before the amendment allowing induction therapy and $152(80.4 \%)$ were enrolled after the amendment. Seventy-three $(38.6 \%)$ of the patients underwent some form of induction chemotherapy and/or radiation therapy. All 73 patients who underwent induction therapy were enrolled after the amendment. Of these, 67 (35.5\% of the total) of the patients underwent preoperative chemotherapy and radiation therapy, $4(2.1 \%$ of the total) underwent preoperative chemotherapy only, and $2(1.1 \%)$ underwent preoperative radiation therapy only.

CT revealed that 97 patients $(51.3 \%)$ were $\mathrm{T} 0, \mathrm{~T} 1$, or $\mathrm{T} 2$ and $92(48.7 \%)$ were T3; $166(87.8 \%)$ were N0 and 23 (12.2\%) were $\mathrm{N} 1$; and 187 (98.9\%) were M0 and $2(1.1 \%)$ were M1a. PET alone showed no abnormal FDG uptake corresponding to the primary tumor in $17(9.0 \%)$ patients and identified the primary tumor in 172 (91.0\%). By PET, 
TABLE 1. Nodal agreement between CT and PET $(n=189)$

\begin{tabular}{lcl}
\hline & PET: N0 & PET: N1 \\
\hline CT: N0 & $121(64.0)$ & $45(23.8)$ \\
CT: N1 & $10(5.3)$ & $13(6.9)$
\end{tabular}

Values are $n(\%)$.

$131(69.3 \%)$ were N0 and 58 (30.7\%) were N1; $154(81.5 \%)$ were M0, 2 (1.17\%) were M1a, and 33 (17.5\%) were M1b. Final histologic type was squamous in $24(12.7 \%)$, adenocarcinoma in $159(84.1 \%)$, carcinoma in $2(1.1 \%)$, and other in $4(2.1 \%)$. Nodal and metastasis agreement between CT and PET are displayed in Tables 1 and 2, respectively.

Table 3 displays the CT by PET findings with the status of the patient regarding confirmatory procedures. There were 9 patients $(4.8 \%$; $95 \%$ CI $2.2 \%-8.9 \%)$ classified as M1b by PET and confirmed as true positive findings. Additionally, there were 18 patients upstaged by PET from M0 to $\mathrm{M} 1 \mathrm{~b}$ in whom confirmatory procedures were not done. Of these 18 patients, 1 patient had rib and pelvis lesions that were still suggestive of M1b disease after confirmatory bone scintigraphy, 1 patient had a liver/right adrenal lesion that was still suggestive after ultrasonography, and the other patients had hilar, cervical, supraclavicular, or other lymphadenopathy that met the criteria for M1b disease. Thirteen of these patients had a surgical resection and 5 did not. Three of the 13 patients undergoing resection despite unconfirmed PET suggestion of M1 disease demonstrated recurrence in the first 6 months. There were 5 patients (2.6\%; $95 \%$ CI $0.9 \%-6.1 \%)$ who apparently were upstaged from M0 to M1b by PET but who had normal or inconclusive confirmatory procedures. Four of these 5 underwent resection and none of them had recurrence or progression in the first 6 months. There was 1 confirmed patient who was upstaged from M0 to M1a by PET. One additional patient apparently was upstaged from M0 to M1a with normal or inconclusive confirmatory procedures suggesting a false positive PET.

Adverse events of grade 3 and above are displayed in Table E-2. One of the costs of routine PET and confirmation of positive findings would be any morbidity incurred by the confirmatory procedures. Seven patients ( $5 \mathrm{M} 0-\mathrm{M} 1 \mathrm{~b}$; $2 \mathrm{M} 0$ M1a) had negative confirmatory procedures. One patient underwent an adrenalectomy for a false positive PET sug-

TABLE 2. Metastasis agreement between CT and PET ( $n=189)$

\begin{tabular}{lccc}
\hline & PET: M0 & PET: M1a & PET: M1b \\
\hline CT: M0 & $153(81.0)$ & $2(1.1)$ & $32(16.9)$ \\
CT: M1a & $1(0.5)$ & $0(0)$ & $1(0.5)$
\end{tabular}

Values are $n(\%)$.
TABLE 3. CT by PET findings with confirmation procedure status $(\mathbf{n}=189)$

\begin{tabular}{lllr}
\hline CT & PET & Confirmatory procedures: & n (\%) \\
\hline M0/M1a & M0 & Not required & $154(81.5)$ \\
M0 & M1a & Confirmed positive & $1(0.5)$ \\
M0 & M1a & Falsely positive PET & $1(0.5)$ \\
M0 & M1b & Not done & $18(9.5)$ \\
M0 & M1b & Confirmed positive & $9(4.8)$ \\
M0 & M1b & Falsely positive PET & $5(2.6)$ \\
M1a & M1b & Falsely positive PET & $1(0.5)$ \\
\hline
\end{tabular}

gesting an adrenal metastasis. This patient experienced the morbidity of the adrenalectomy surgery as well as the need for subsequent therapy for adrenal insufficiency. Another patient incurred a grade 3 adverse event for a wound complication after a confirmatory procedure for a false positive PET.

A total of $145(77.5 \%)$ patients went on to have surgery, $42(22.5 \%)$ did not, and in 2 patients the surgical status cannot be determined. Analysis of resection status, stratified on the use or nonuse of induction therapy, showed a resection rate of $68 \%$ in the patients given induction therapy and a resection rate of $83 \%$ in those not given induction therapy $(P=.014)$. The reasons for no resection in evaluable patients included the following: M1 disease found by PET and confirmed (9), M1 disease found by PET and not confirmed (2), M1 disease in the liver, peritoneum, or lung discovered at exploration but not found by PET (7), decline or death before surgery (10), patient refusal of surgery (7), unresectable local tumor at exploration (5), and extensive N1 disease precluding operation (2). Three of the patients who underwent resection died perioperatively and $8(5.6 \%)$ of the remaining 142 patients undergoing resection had a relapse of their cancer within 6 months of registration to the trial.

There were 39 patients who were classified as ineligible for analysis for the primary objective of the trial because the findings of the conventional staging evaluation suggested metastasis. The findings in these patients included lung nodules (22), liver nodules (13), adrenal masses (4), M1 lymph nodes (3), bone lesions (2), ascites, pleural effusion, and a suggestive inguinal mass. Some patients had more than one abnormality on CT, so the total exceeds 39 . Of these patients, 20 subsequently underwent resection, $15 \mathrm{did}$ not undergo resection, and the resection status of the remaining 4 is unknown. The 15 patients who did not undergo resection include 12 who had evidence of metastasis that had been identified by the CT scan and confirmed by PET and other subsequent tests. The other 3 patients include 1 who refused surgery for unstated reasons, 1 who declined surgery after a complete clinical response to induction therapy, and 1 who was offered photodynamic therapy as an 
alternative to surgery. In the 20 patients who underwent resection despite evidence of metastasis on CT scan, many had PET results that contradicted the CT and thus the PET provided encouragement to consider surgical resection in the face of CT findings suggestive of metastatic disease. Four (20\%) of these 20 had a recurrence within the first 6 months of resection, in contrast to the $5.6 \%$ recurrence rate at 6 months seen in those patients eligible for the trial.

\section{Discussion}

The primary objective of this trial was to evaluate the usefulness of adding PET to the staging of patients with resectable esophageal cancer after completion of routine staging procedures. This trial therefore differs from most other series in which incomplete conventional staging was performed. The study design determined at the outset that a $5 \%$ or lower rate of distant metastasis detection would be considered inconsequential (the null hypothesis) and a greater than $10 \%$ rate of metastasis detection would be considered clinically important (the alternative hypothesis). The actual rate of detection of metastasis, confirmed by biopsy, was $4.8 \%$. However, in an additional $9.5 \%$ of patients with apparent PET-detected metastases, the PET findings were accepted by the treating surgeon as evidence without the required confirmation. Accordingly, the overall rate of detected metastases may be as high as $4.8 \% \pm 9.5 \%$ or $14.3 \%$ (95\% CI: $9.6 \%-20.1 \%$ ). It is unlikely that the actual fraction of true positive PET findings is at the high end of the range, since many of the unconfirmed findings were noted in patients who subsequently underwent surgical resection and had no evidence of recurrence or progression at 6 months.

Limitations of this trial are important to consider. Technology has advanced since this trial was opened in 1999, and the current widespread use of integrated PET/CT could alter the yield of PET beyond that seen in this study. The large number of ineligible patients enrolled in this study led to a number of exclusions that were not anticipated by the study design. It is possible that the exclusion of such patients made the enrollment criteria more rigorous than those adhered to in actual clinical practice, thereby decreasing the frequency of true positive metastatic lesions detected by PET. Assessment of this dual purpose of PET, for detecting unsuspected metastasis and also for confirming or excluding metastasis suspected on the basis of CT findings, was not part of the intended scope of this trial.

Although it seems obvious that allowing induction therapy in a trial such as this would alter the interpretation of the results, it is not obvious how such a change would alter the measured value of PET in this setting. If a falsely PET negative, but actually positive M1a celiac node was converted to pathologically negative, then induction therapy would mask a weakness of PET by making it appear correct when it is not. If a truly positive M1a node was positive on PET but was converted to negative by induction therapy, it would be scored as a false positive and it would make PET look worse. Thus, the direction and the severity of the bias are unknown, but the presence of bias is suspected.

Additionally, the impact of endoscopic ultrasonography was not anticipated at the outset of this trial and is therefore difficult to incorporate into the analysis and interpretation of these results. The use of endoscopic ultrasonography was sporadic in the early patients enrolled, but became more commonplace toward the end of accrual. Positive findings resulting from endoscopic ultrasonography, such as the T3, N1, or M1a stage descriptors, would often lead to an apparent change in treatment (ie, induction therapy) even in the presence of a Tx N0 M0 PET report.

The main assumptions when this trial was developed were that M1b disease would absolutely contraindicate surgery and that M1a disease would be a strong relative contraindication to surgery. Therefore, the anticipated impact of the PET findings on the surgical treatment of the enrolled patients was believed to depend on the rate of detection of M1a and M1b findings. In the analysis, it became apparent that several patients did not undergo resection after PET demonstrated multistation N1 disease. It is therefore possible that the impact of PET on surgical decision making goes beyond the detection of M1 disease. In addition, when the study was amended to allow induction therapy between PET and surgery, the analysis was further complicated by the fact that the PET finding of N1 nodal disease could directly or indirectly lead to the selection of an induction therapy strategy. Because a substantial number of patients treated with induction therapy never undergo subsequent surgical resection, it is possible that the impact of PET on surgical resection rates has been underestimated in this study.

A potential downside to routine use of PET for staging in these patients is the burden imposed by the false positive PET findings. Assuming that all positive findings would be confirmed, a false positive PET result might lead to more invasive procedures and therefore might result in cost and morbidity beyond that attributable to the PET procedure itself. An example in this trial is an individual patient with apparent M1 disease on PET who underwent an adrenalectomy. The adrenal was resected and shown to be normal, the PET was thus falsely positive, and the patient required therapy for adrenal insufficiency. Because the positive PET findings in this study were not all properly assessed by confirmatory studies in accordance with the trial protocol, the risks and costs that characterize the burden of false positive results are likely underestimated here.

One interpretation of our results is that PET for esophageal cancer has many attributes, only one of which is the detection of M1 disease in patients otherwise believed to be surgical candidates after routine CT staging. PET can be 
used as a confirmatory test when there are indeterminate abnormalities on CT. There is a growing body of literature suggesting that the standardized uptake value for FDG in the primary tumor, a semiquantitative measure of the tumor's glucose metabolism, may help identify patients at elevated risk for recurrence and may, therefore, identify appropriate patients for induction therapy or adjuvant therapy. ${ }^{6}$ Another body of literature addresses the hypothesis that PET, obtained during or after induction therapy, can predict pathologic response and identify subsets of patients with better and worse prognoses. ${ }^{7-12}$ None of these specific indications was tested by the current trial.

In summary, FDG-PET in patients with esophageal cancer without evidence of metastasis after conventional workup identified unsuspected distant metastatic disease in at least 4.8\% (95\% CI: $2.2 \%-8.9 \%$ ) of cases. An additional 3.7\% (95\% CI 1.5\%-7.5\%) had unconfirmed evidence of M1b disease and were treated nonsurgically, at least in part owing to the PET findings. In most cases, these PET-detected metastases should be confirmed before excluding a patient from surgical consideration, since apparent M1 findings by PET in at least 3.7\% (95\% CI: $1.5 \%-7.5 \%$ ) were false positives. An additional 5\% of patients can be expected to harbor metastatic disease that escapes detection by both CT and PET.

\section{References}

1. Flanagan FL, Dehdashti F, Siegel BA, Trask DD, Sundaresan SR, Patterson GA, et al. Staging of esophageal cancer with 18Ffluorodeoxyglucose positron emission tomography. AJR Am J Roentgenol. 1997;168:417-24.

2. Block MI, Patterson GA, Sundaresan RS, Bailey MS, Flanagan FL, Dehdashti F, et al. Improvement in staging of esophageal cancer with the addition of positron emission tomography. Ann Thorac Surg. 1997;64:770-6; discussion 776-7.

3. Luketich JD, Schauer PR, Meltzer CC, Andreneau RJ, Urso GK, Townsend DW, et al. Role of positron emission tomography in staging esophageal cancer. Ann Thorac Surg. 1997;64:765-9.

4. van Westreenen HL, Westerterp M, Bossuyt PMM, Pruim J, Sloof $\mathrm{GW}$, van Lanschot JJ, et al. Systematic review of the staging performance of $18 \mathrm{~F}$-fluorodeoxyglucose positron emission tomography in esophageal cancer. J Clin Oncol. 2004;22:3805-12.

5. Green SJ, Dahlberg S. Planned versus attained design in phase II clinical trials. Stats Med. 1992;11:853-62.

6. Hong D, Lunagomez S, Kim EE, Lee JH, Bresalier RS, Swisher SG, et al. Value of baseline positron emission tomography for predicting overall survival in patient with nonmetastatic esophageal or gastroesophageal junction carcinoma. Cancer. 2005;104:1620-6.

7. Downey RJ, Akhurst T, Ilson D, Ginsberg R, Bains MS, Gonen M, et al. Whole body 18FDG-PET and the response of esophageal cancer to induction therapy: results of a prospective trial. J Clin Oncol. 2003;21:428-32.

8. Swisher SG, Maish M, Erasmus JJ, Correa AM, Ajani JA, Bresalier R, et al. Utility of PET, CT, and EUS to identify pathologic responders in esophageal cancer. Ann Thorac Surg. 2004;78:1152-60; discussion 1152-60.

9. Song SY, Kim JH, Ryu JS, Lee GH, Kim SB, Park SI, et al. FDG-PET in the prediction of pathologic response after neoadjuvant chemoradiotherapy in locally advanced, resectable esophageal cancer. Int $J$ Radiat Oncol Biol Phys. 2005;63:1053-9.

10. Cerfolio RJ, Bryant AS, Ohja B, Bartolucci AA, Eloubeidi MA. The accuracy of endoscopic ultrasonography with fine-needle aspiration, integrated positron emission tomography with computed tomography, and computed tomography in restaging patients with esophageal cancer after neoadjuvant chemoradiotherapy. $J$ Thorac Cardiovasc Surg. 2005; 129:1232-41.

11. Brucher BL, Weber W, Bauer M, Fink U, Avril N, Stein HJ, et al. Neoadjuvant therapy of esophageal squamous cell carcinoma: response evaluation by positron emission tomography. [see comment]. Ann Surg. 2001;233:300-9.

12. Kato H, Kuwano H, Nakajima M, Iyazaki T, Yoshikawa M, Masuda $\mathrm{N}$, et al. Usefulness of positron emission tomography for assessing the response of neoadjuvant chemoradiotherapy in patients with esophageal cancer. Am J Surg. 2002;184:279-83.

\section{Discussion}

Dr Mark J. Krasna (Baltimore, Md). First, I wish to congratulate Dr Meyers on an excellent presentation. Following up on the pioneering work at Washington University on PET scanning for thoracic malignancies, the ACSOG undertook two clinical trials to determine the role of PET scanning in our specialty. After presenting their findings from Z40 on PET scanning for lung cancer, the ACOSOG surgeons are now presenting the results of the first multi-institutional prospective phase 2 trial for PET scanning in esophageal cancer to put this new modality in perspective. All the surgeons who undertook this goal are to be congratulated for their completion of this trial. This in itself is a great accomplishment and is an example to us all to study questions in thoracic surgery thoroughly by clinical trial wherever possible.

The objective of this study was to see whether PET scan avoided unnecessary surgery in $5 \%$ or more of patients with esophageal cancer. The primary result of the study may be summarized as follows: PET scan identified suggestive M1 disease, including M1a and M1b, in 11 patients, or 4.8\%. PET scan missed M1 disease in 7 patients who underwent exploration. Also, PET scan found that $31 \%$ of the patients had N1 disease, including extensive N1, which sometimes led to surgeons avoiding surgery. This is a little lower than expected for most surgical series, in which almost two thirds of patients are found to have N1 disease.

Interestingly, this study also provides a snapshot of the current practice among CT surgeons in managing esophageal cancer. Over $38 \%$ of the patients were referred for induction chemoradiation therapy in this study. This adds to the recent "Patterns of Care Survey" data from the Radiation Therapy Oncology Group that show the inroads that surgeons have made in incorporating chemoradiation and surgery in the treatment algorithm for esophageal cancer, and it is supported by recent phase 2 and phase 3 studies. Unfortunately, this also limits the interpretation of the results, as it would have been best to compare PET to pathologic findings in patients who were undergoing more surgery alone. I have three questions.

First, how have you defined the role of surgery in patients with M1a disease? On what basis do you decide not to perform resection in these patients and those with extensive N1 disease? Second, what is the average cost of PET scanning? Do these results really justify using this huge outlay in expenditure, in your opinion? Finally, although there were 262 patients who were enrolled, the ineligibility and unevaluable rate was quite high, much higher than you expected. Given the borderline significance of these findings, do the authors think that the results would be different, and how would you suggest to us the take-home message be interpreted? In other words, what is the current role of PET scan in our practice in esophageal cancer? 
Dr Meyers. Thanks for your questions, Dr Krasna. Your first question asked about the role of surgery in M1a, and this trial actually left it to the surgeons to make a decision about how to deal with their patients once they have had the PET and CT staging. If I were to answer your question, it would represent my personal strategy for M1a, and it includes very limited surgery. I would offer M1a patients surgery only if they had no evidence of distant metastatic disease and if they underwent induction therapy and were good surgical candidates afterward. However, that question was not addressed by this study in any way at all.

The average cost of a PET scan is around $\$ 2000$. We did not have a corollary cost-effectiveness analysis planned when this study was initiated, but I am sure that we could use these data to come up with an analysis to look at the benefit and cost associations with PET scanning. I am sure that that would be a natural follow-on to this study. PET scans cost \$2000 apiece, and when you see that it only changed the stage-directed use of surgery in only $5 \%$ to $8 \%$ of the patients, cost is certainly a consideration.

Finally, you asked about the question of ineligible patients and the fallout from the number of patients enrolled to the number of patients who were actually analyzed and how those results might differ. Certainly the screened cohort, before and after exclusions, is going to differ in that there will be a decrease in the apparent yield of "new" M1 disease by the PET scan. However, I think that the exclusions offer as close as to a real estimate of the value of PET scan that we could report. I think in the single-center studies, including our own, the enrollment was not as carefully scrutinized as it was in these patients, and we found that there were quite a number of patients with borderline CT abnormalities in whom the PET scan was used to confirm an abnormal CT finding rather than to look for something that was undetected by the CT scan. Those are two different goals. The point of this study was to look at new detection of otherwise unsuspected metastatic disease.

\section{JTCVS On-Line Manuscript Submission and Review}

The Journal of Thoracic and Cardiovascular Surgery requires authors and reviewers to submit all new and revised manuscripts and reviews via Editorial Manager. Point your browser to http://jtcvs.editorialmanager.com, log in as author or reviewer (as appropriate), and follow the instructions provided.

To retrieve your username and password, click "Forget your password?" on the Editorial Manager log-in page.

If you have questions or experience problems uploading your manuscript or review, please contact the editorial office:

Telephone: 215-762-1854

E-mail: jtcvs@drexelmed.edu 


\section{TABLE E1. Enrolling institutions}

Akron General Hospital
Allegheny Cancer Center
Baylor University Medical Center
Duke University Medical Center
Good Samaritan Hospital
Jameson Hospital
Latter Day Saints Hospital
Massachusetts General Hospital
Medical College of Virginia
Medical University of South Carolina
Memorial Sloan-Kettering Cancer Center
Ohio State University, Arthur G. James Cancer Hospital
Providence Portland Medical Center
Stanford University
State University of New York, Upstate Medical University
Swedish Medical Center
University of Alabama at Birmingham
University of Colorado
University of Maryland
University of Tennessee
Washington University Medical Center
Westmoreland Hospital
William Beaumont Hospital

TABLE E2. Adverse events grade 3 and above

\begin{tabular}{|c|c|c|c|}
\hline Adverse events & Grade 3 & Grade 4 & Grade 5 (death) \\
\hline Nausea & $1(0.5)$ & & \\
\hline Effusion, pleural & $2(1.1)$ & & \\
\hline Dehydration & $1(0.5)$ & & \\
\hline Pancreatitis & $1(0.5)$ & & \\
\hline Pneumonitis & $1(0.5)$ & $1(0.5)$ & \\
\hline $\begin{array}{l}\text { Adult respiratory distress } \\
\text { syndrome }\end{array}$ & $1(0.5)$ & $1(0.5)$ & \\
\hline Arrhythmia & $1(0.5)$ & & \\
\hline Ischemia/infarction & $1(0.5)$ & & \\
\hline Pain, abdominal & $1(0.5)$ & & \\
\hline $\begin{array}{l}\text { Infection, no antigen } \\
\text { neutralizing capacity }\end{array}$ & $1(0.5)$ & & \\
\hline Thrombocytopenia & $1(0.5)$ & & \\
\hline Fistula, esophagus & $1(0.5)$ & $1(0.5)$ & \\
\hline Hyperglycemia & $2(1.1)$ & & \\
\hline Pneumothorax & $1(0.5)$ & & \\
\hline Wound, noninfectious & $1(0.5)$ & & \\
\hline Hemorrhage & $1(0.5)$ & & \\
\hline Febrile neutropenia & $1(0.5)$ & & \\
\hline Infection & $2(1.1)$ & & \\
\hline Neurologic & $1(0.5)$ & & \\
\hline Pain & $1(0.5)$ & & \\
\hline Pulmonary & $2(1.1)$ & $1(0.5)$ & \\
\hline Diaphragm rupture & $1(0.5)$ & & \\
\hline Dysphagia & & $1(0.5)$ & \\
\hline Leukopenia & & $1(0.5)$ & \\
\hline Thrombosis & & $1(0.5)$ & \\
\hline $\begin{array}{l}\text { Arrhythmia, supraventricular } \\
\text { tachycardia }\end{array}$ & & $1(0.5)$ & \\
\hline Acidosis & & $1(0.5)$ & \\
\hline Apnea & & $1(0.5)$ & \\
\hline Endocrine & & $2(1.1)$ & \\
\hline $\begin{array}{l}\text { Infection, no antigen } \\
\text { neutralizing capacity }\end{array}$ & & $1(0.5)$ & $2(1.1)$ \\
\hline Hematemesis & & & $1(0.5)$ \\
\hline
\end{tabular}

Values are $\mathrm{n}(\%)$. 